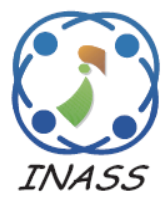

\title{
Multi-Channel Convolutional Neural Network for Prediction of Leaf Disease and Soil Properties
}

\author{
Padmapriya Dhandapani ${ }^{1 *}$ \\ Arulmozhi Varadarajan ${ }^{1}$ \\ ${ }^{I}$ Department of Research - Ph.D Computer Science, Tiruppur Kumaran College for Women, \\ Tiruppur, Tamil Nadu, India \\ * Corresponding author’s Email: jeattn2000phd@gmail.com
}

\begin{abstract}
Prediction of leaf disease and soil property helps farmers improve crop production quality through maintaining the soil property and taking proper actions for leaf disease. Various techniques have been developed to predict leaf disease and soil properties. Support Vector Machine (SVM) is one of the machine learning techniques that was used to predict leaf disease and soil properties. To predict the leaf disease and soil property, SVM processed the extracted features from leaf images and soil images. Deep learning can be used for prediction, which has the advantage of machine learning is that one does not need to be concerned about domain knowledge as no feature engineering is required in this, unlike SVM-based prediction. In addition to this, SVM-based prediction is not very effective for handling multiple inputs. A Convolutional Neural Network (CNN) is a deep learner which was applied for the prediction of leaf disease and soil property. Even though this method has better performance, the information from leaf and soil images is mixed together, which may affect the prediction accuracy. So, in this paper, a Multichannel CNN (MCNN) method is introduced in which individual channels are used for leaf and soil images. In MCNN, the feature learning using MCNN for leaf images is kept distinct from the soil image to avoid data fusion between the leaf and soil images. The features related to leaf and soil images are paired and transferred over the corresponding channels for the prediction of leaf disease and soil property. After the prediction of leaf disease and soil property, the correlation between leaf disease and soil property is identified using the Pearson correlation coefficient and it is sent to the farmers using mobile phones to improve their crop production. Finally, these methods are validated by using different leaf infections and soil images for 3 types of crops. The experimental results show that the MCNN method achieves an average accuracy of $87.77 \%$ for leaf disease prediction and $90.38 \%$ for soil property prediction compared to the classical methods.
\end{abstract}

Keywords: Leaf disease prediction, Soil property prediction, Deep learning, Multi-channel convolutional neural network.

\section{Introduction}

In cultivation, plants' leaves are essential for obtaining data regarding the nature and quantity of horizontal yield. Various aspects influence the production of crops, such as soil infertility, climate change and the presence of weeds. In contrast, leaf disease is a global hazard and a cause of financial damage for the development of many agricultural products. Precision farming [1-3] makes use of cutting-edge technologies to optimize crop production decisions making can be achieved by visual inspections by experts. However, it is normally time-consuming and highly expensive. In this era of technology and automation, it is not a very efficient approach. It would be much better if an automated system was used to predict the leaf disease automatically [4].

An automated system depending on SVM was developed for predicting the leaf disease with the consideration of different soil properties.

Initially, leaf and soil images were captured and sent to the server using Internet-of-Things (IoT) techniques. The texture features and contour-based shape descriptors were extracted from the leaf 
images. Also, the color histogram-based features were extracted from soil images. These features were used by SVM for plant leaf disease prediction. Deep learning is used for forecasting plant leaf disease. The benefit of deep learning over machine learning is that one does not need to be concerned about domain knowledge as no feature engineering is required in this, unlike in SVM-based leaf disease prediction. Also, SVM-based leaf disease prediction is not very effective for handling multiple inputs.

$\mathrm{CNN}$ is a deep learning technique that was used for leaf disease prediction. Taking leaf and soil images as input, $\mathrm{CNN}$ processes the input on convolutional, pooling and fully connected layers to predict the leaf disease. In CNN-based leaf disease prediction, the information from soil and leaf images are mixed, which may affect the prediction accuracy. So, in this paper, an MCNN is introduced where separate channels are used for leaf and soil images using the concept that the primary attribute training using $\mathrm{CNN}$ for leaf image is being preserved regarding soil image to avoid the primary data fusion amid leaf and soil image. Hence, the features corresponding to each leaf and soil images are paired accordingly and transferred over the corresponding channels for leaf disease prediction. It also guarantees optimum generalizability and identifies the complex information in leaf and soil images for better prediction of leaf diseases and identification of correlations between leaf disease and soil properties.

The remaining part of this article is structured as follows: Section 2 is an examination of the research related to the prediction of leaf disease. Section 3 explains the MCNN for leaf disease prediction and Section 4 demonstrates its efficiency. Section 5 summarizes the paper with future scope.

\section{Literature survey}

Dhingra et al. [5] proposed a computer visionbased neutrosophic method to identify leaf infection. Initially, the collected image was segmented based on fuzzy logic and the segmented region was differentiated as proper, improper and transitional areas. According to these areas, novel attribute subsets were evaluated for identifying the leaf as a fit or infected leaf. However, the selection of membership function in fuzzy logic greatly influences the performance of leaf disease identification.

Pantazi et al. [6] proposed an automatic leaf disease detection technique by using an image feature analysis technique and one class of classifiers. For feature extraction, Local Binary
Patterns (LBPs) were applied to the collected images. The extracted features were trained and tested in one classifier for leaf disease detection. However, the symptoms in some cases were detected as outliers for other diseases concerning the same crop. Geetharamani \& Pandian [7] used Principal Component Analysis (PCA) and deep CNN for the identification of plant leaf diseases. Some finetuning techniques will be used to enhance the accuracy of this model.

Jiang et al. [8] developed a model relying on Improved CNN (ICNN) for recognizing apple leaf infections. However, it is difficult when the infected region engages only a small segment of the image. Sorte et al. [9] proposed a coffee leaf infection detection method depending on the deep learner and texture features. More texture features like spectral features need to be extracted and enhanced to enhance the performance of this recognition method.

Saleem et al. [10] designed three different deep learner meta-architectures such as Region-based Fully Convolutional Networks (RFCN), Single Shot multi-box Detector (SSD) and Faster Region-based CNN (RCNN) were explored through the TensorFlow object detection framework for identifying diseases in plant species. However, it needs further improvement in terms of mean average precision.

Nigam et al. [11] developed a new idea for the recognition and classification of paddy leaf diseases. First, different paddy leaves were collected as digital images and then the RGB model was transformed into a Hue, Saturation, Value (HSV) model to resize the image by applying k-means clustering with image segmentation. The particular features in the transformed model were extracted by using PCA and these features were used in the Bacterial Foraging Optimization-Deep Neural Network (BFO-DNN) classifier for paddy leaf disease recognition and classification. However, the recognition time was high and DNN was computationally expensive while increasing the network depth.

Sethy et al. [12] proposed a technique based on deep CNN for the identification of rice leaf disease. The deep features in the collected images were extracted by using deep CNN architectures and the extracted features were given as input to SVM for identification of rice leaf disease. A more fine-tuned CNN model will be used in the future with the expectation of better performance. Sun et al. [13] used the CNN technique for the detection of northern maize leaf blight in a complex field environment. Because of less convolution, this technique returned lower semantics and more noise, which affected the performance of the detection of 


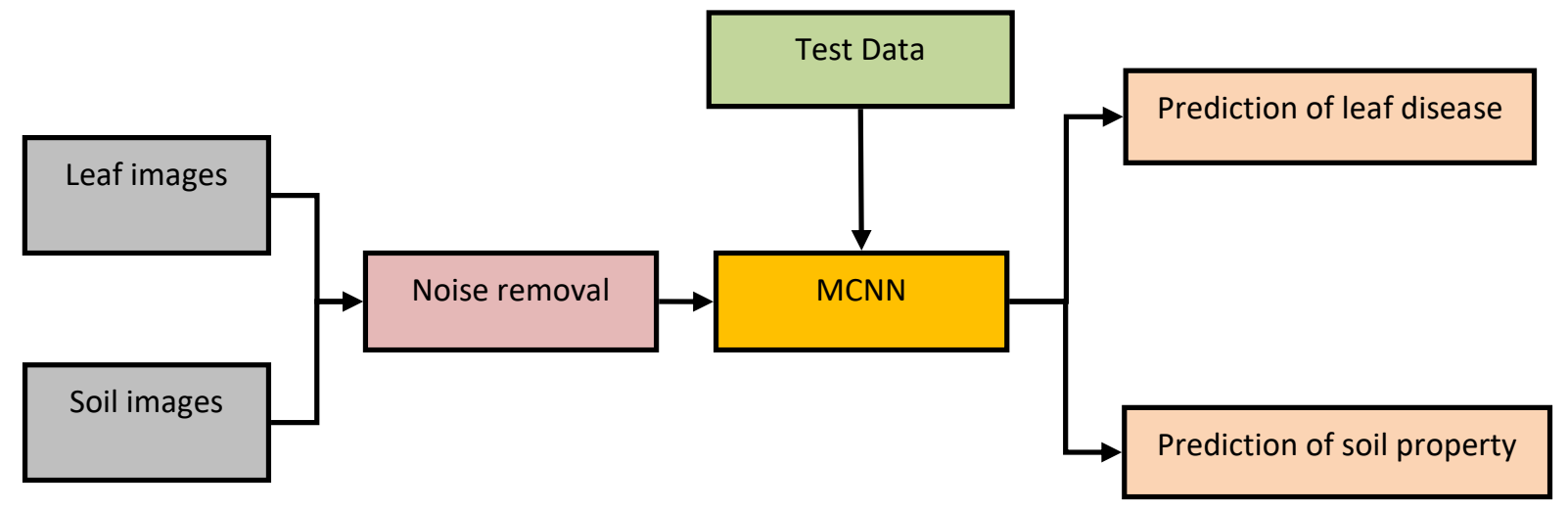

Figure. $1 \mathrm{MCNN}$-based leaf disease prediction and soil property prediction

northern maize leaf blight.

\section{Proposed methodology}

This part describes the MCNN-based leaf disease prediction in detail. Initially, plant leaf images, as well as soil images, are collected by using a digital camera. The captured images are transmitted through either a wired or wireless network to an image processing unit for further processing. After the collection of leaf and soil images, a noise removal technique is applied to the collected images for the pre-processing process. The pre-processed image is given as input to the MCNN, which allows leaf and soil images to transfer via two dissimilar channels to optimize the attribute training and offer the forecasting possibilities through aggregating the attribute maps of the two channels. Based on the probability value, the correlation between diseased leaves and soil properties is predicted. Fig. 1 shows the block diagram of the MCNN-based leaf disease prediction method.

\subsection{Data collection}

First, the soil images and their related leaf disease images are collected for three different plants, like cotton, pineapple and strawberry. The considered types of soil images are over-watering and high nitrogen soil, contaminated soil, damp soil, high humidity soil, warm overlay moist soil and warm heated soil. Among these types, overwatering and high-nitrogen soil cause mealybugs on plant leaves. The warm heated soil causes spider mite disease and the warm overlay moist soil causes Rhizoctonia disease. The cylindrocladium leaf disease is caused by the high humidity soil and thielaviopsis disease (black root rot) is caused by the damp soil at a temperature of $55-65^{\circ} \mathrm{F}$. Also, ralstonia solancearum (bacterial wilt) leaf disease is caused by contaminated soil. Normally, the collected images are corrupted by different types of noises. So, it is needed to remove the noise from the images for better prediction.

\subsection{Noise removal}

An image (leaf or soil image) is assumed as a lattice pattern of $2 \mathrm{D}$ cell automata wherein every cell $\left(x_{0}, y_{0}\right)$ relates to the image pixel $(x, y)$ and probable conditions of the cells are the certain color value of that pixel. Every cell communicates with its adjacent pixels and changes its condition by the switch factor. Assume $\left\{I_{1}(t), I_{2}(t), \ldots, I_{n}(t)\right\}$ is the collection of $n$ pixels that are measured in a Moore vicinity of the pixel at time $t$. The Moore vicinity of distance $r$ is represented as:

$$
N\left(x_{0}, y_{0}\right)=\left\{(x, y):\left|x-x_{0}\right| \leq r,\left|y-y_{0}\right| \leq r\right\}
$$

In Eq. (1) $N$ represents neighbor of pixel and $\mathrm{x}, \mathrm{y}$ represent pixel of the image. The order statistics result is given by ordering these values in increasing order of their intensity and denoted as $\left\{I_{i 1}(t), I_{i 2}(t), \ldots, I_{i n}(t)\right\}$. The centered pixel alters its values at the time $t+1$ is given as:

$$
\begin{gathered}
I(t+1)=\frac{1}{n-2(\alpha n)} \sum_{j=(\alpha n)+1}^{n-(\alpha n)} I_{j}(t), \\
0 \leq \alpha<0.5
\end{gathered}
$$

In Eq. (2), $\mathrm{n}$ represents the number of neighbor pixels and $\alpha$ represents the scaling factor. The maximum and minimum intensity values are eliminated and the average of remaining pixel intensity values is calculated. Also, the quality of soil and leaf image is improved by using the histogram equalization technique which eliminates the background information, redundant and hidden 
details.

\subsection{Plant leaf disease and soil property prediction}

After the noise removal from leaf and soil images, it is given as input to MCNN for plant leaf disease and soil property prediction. The model architecture for the MCNN is shown in Fig. 2. The MCNN architecture has two input channels for leaf and soil images, respectively. The features corresponding to each leaf and soil image are paired accordingly and transmitted over the corresponding channels.

The convolution layer is the most unique part of CNN that is applied to traverse over the image matrix for generating an ultimate attribute matrix of spatially-oriented attributes by adapted filtering.
This adapted filtering for the convolution layers in the several channels is regulated with respect to the input shape of the image matrix. The best profile for the filtering is selected by

$$
f(x, 1)=\left[\text { round }\left(\frac{x}{2}\right)+x \odot 2\right]
$$

In Eq. (3), $x$ denotes the number of leaf or soil images used for training, $(x, 1)$ denotes the profile of the filtering, round $(\cdot)$ denotes the round function and $\odot$ represents the modulo function. Eq. (3) takes the leaf or soil image used for training as input and this image undergoes the addition of a round factor over $x$ and a modulo factor over $x$. It

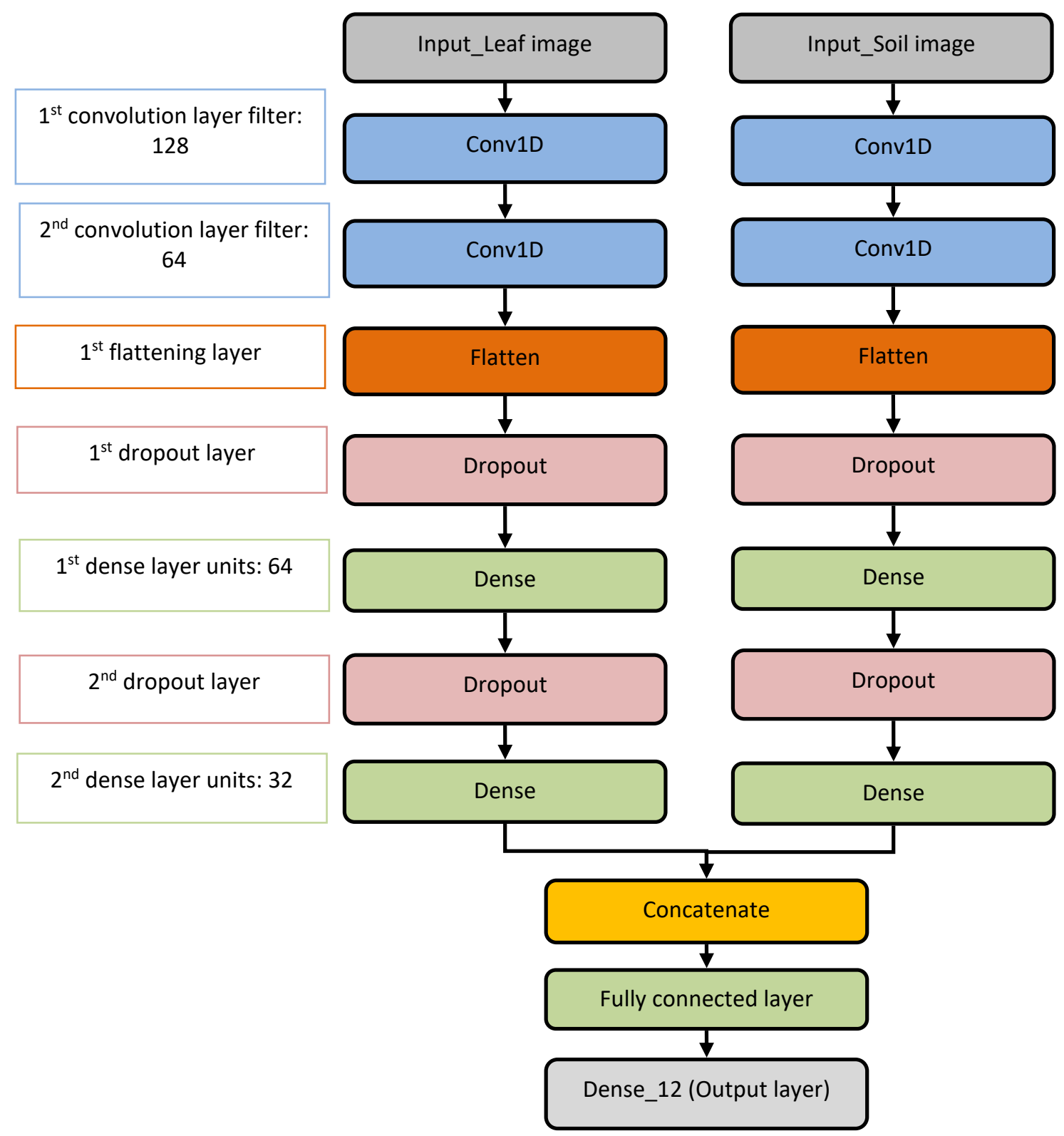

Figure. $2 \mathrm{MCNN}$ architecture for plant leaf disease prediction and soil property prediction 
offers the profile of the adapted filtering. Here, 1-D convolution is used so the profile of the filter is in the format of $(x, 1)$.

The attribute maps from the primary convolution layer are transmitted to the $2^{\text {nd }}$ convolution layer with no sub-sampling process. With the consideration of the large spatial density of the image which is learned on the CNN structure, it is stated that a sub-sampling process like pooling among successive CNN layers reduces the computational cost for prediction. However, the utilization of sub-sampling for the image whose statistical importance is highly vital compared to the spatial configuration possesses data failure. In the prediction of plant leaf disease and soil property, spatial arrangement information is more important. So, in the MCNN architecture, the sub-sampling process has been avoided.

The feature matrix which is generated by the $2^{\text {nd }}$ convolution layer is given as input to the flattening layer. It transforms the attribute matrix from a 2D matrix to a 1D array as the following phases encompass the dense layers. The data must be in one-dimensional format when the set of images is fed to the dense layer.

The outcome of the flattening layer is given as input to the dropout layer, which is typically applied to execute the normalization and support the MCNN to prevent overfitting. It enables MCNN to extract highly complicated and strong feature correlations from leaf and soil images by reducing the number of neurons from the perceptible and hidden layers for performing highly randomized attribute training.

The sixth layer in the MCNN structure is a dense layer which is a fully connected layer with 64 neurons. It performed a linear function on the attribute matrix which is created by the convolution layer. In addition to this, as the convolution layer operates in the vicinity of the spatial collection of distinct filters that move over the image matrix, the dense layer is performed as a global layer in which every layer's node contributes and is linked to each other node in the subsequent layers. Hence, the dense layer in MCNN establishes a global correlation among the attributes and also considers the abstraction of highly complicated structures in the leaf and soil images.

The ninth layer in the MCNN is the concatenation layer, which combines the attribute matrices from every channel. Based on multiple features from leaf and soil images, attribute matrices are aggregated to predict leaf disease and soil property. Hence, for achieving a better prediction of leaf disease and soil properties based on leaf and soil images, the aggregation of attribute matrices between two different channels is needed.

The following layer is the concatenation layer, the successive layer is the fully connected layer with 32 units. It is used to fetch the combined correlations among the aggregated attribute matrices from several channels. It gives the complicated attributes, correlations and structures amid the aggregated attribute matrices which help us predict leaf disease and soil property. The last layer (output) consists of 6 units for 6 different leaf diseases and their related soil properties. The last dense layer gives the forecasting chance of every image for 6 different labels.

After the prediction of leaf disease and its related soil property, a loss function is applied for estimating the variability amid the estimated outcome $(\hat{y})$ and the underlying range $(y)$. In this model, a softmax loss i.e., the cross-entropy loss function is used which compares each predicted class value to the original class value for calculating the score. Then, this is considered for penalizing the prediction chance depending on the variance from the original value. So, the softmax loss is defined by

$$
L(y, \hat{y})=\frac{1}{n} \sum_{i=1}^{n} \sum_{j=1}^{6} L\left(y_{i, j} \log \left(\hat{y}_{i, j}\right)\right)
$$

In Eq. (4), the dual sum is applied on $i^{t h}$ image samples range between 1 and $n$ and the labels vary between 1 and 6 . Also, $y_{i, j}$ is the original class value at $i^{\text {th }}$ image of $j^{\text {th }}$ type and $\hat{y}_{i, j}$ is the prediction for the images as $i^{\text {th }}$ image. Thus, the soil property related to plant leaf diseases is effectively predicted for different types of plants.

\subsection{Communication phase}

The communication phase is used to maintain the soil property and prevent leaf disease by notifying the leaf diseases based on the soil categories through mobile phones to the cultivators. So, it enhances the crop yield by maintaining the soil properties and preventing leaf diseases.

\section{Result and discussion}

In this section, the performance of both leaf disease prediction and soil property prediction is evaluated for existing and proposed methods with different evaluation metrics. In this experiment, different soil images and their related leaf diseases for cotton, pineapple and strawberry plants are collected (described in Section 3.1). The results of MCNN-based leaf disease prediction are compared with the SVM, CNN, fuzzy logic [5], ICNN [8] and RFCN [10] methods. The results of MCNN-based 
soil property prediction are compared with the Logistic Regression (LR), Linear SVM (LSVM), Gaussian SVM (GSVM) [14] and Extreme Learning Machine (ELM) [15] methods. Fig. 3 displays the sample leaf images for each type of disease and Fig. 4 displays the sample soil images for different categories.

\section{Bacterial flight \\ Cylindrocladium}

Mealy bugs

Rhizoctonia

Spider mites

Thielaviopsis

Cylindrocladium

Mealy bugs
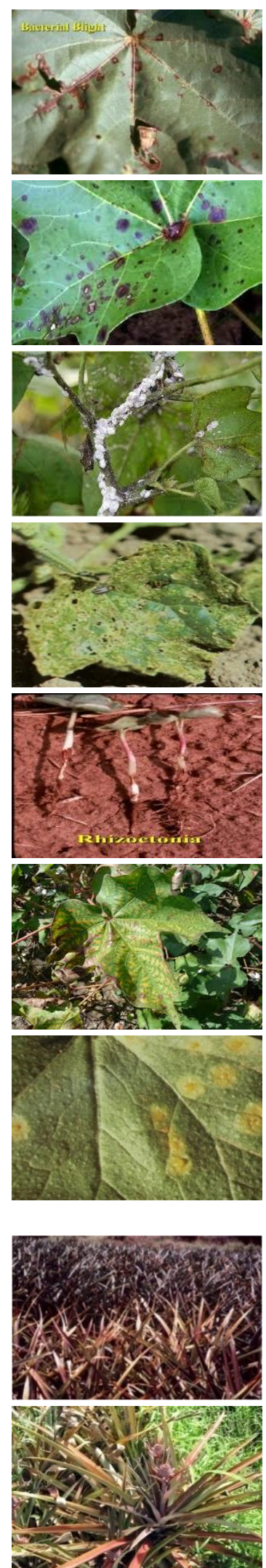

Cotton
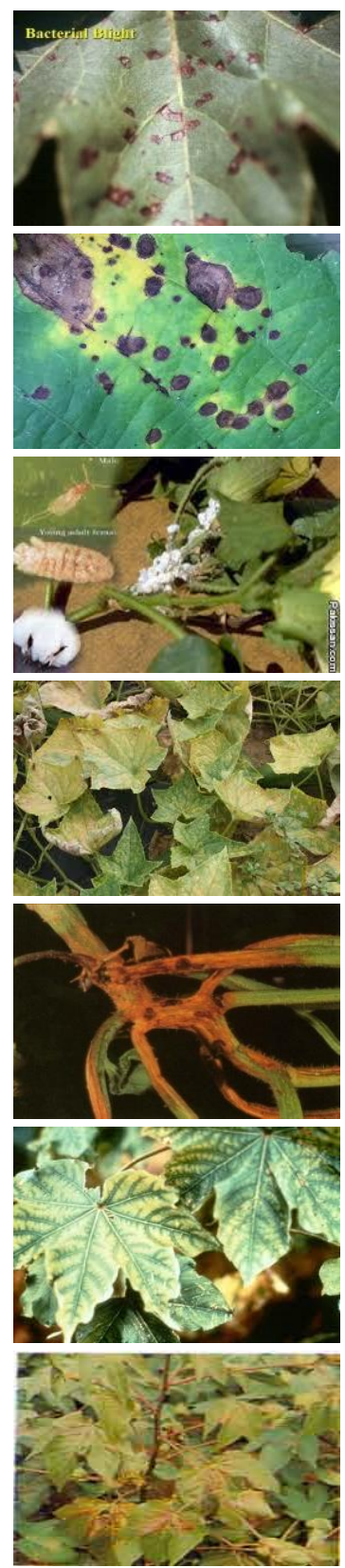

Pineapple

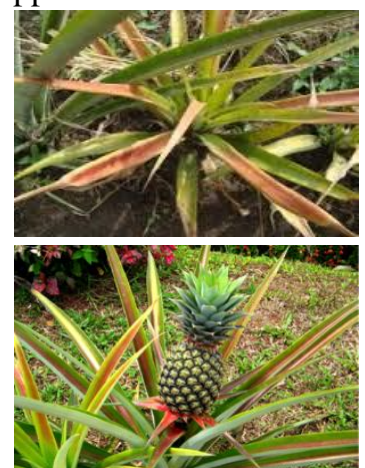

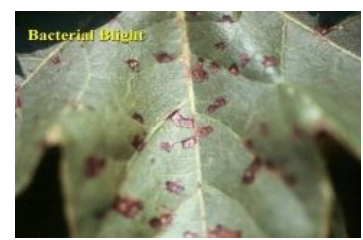
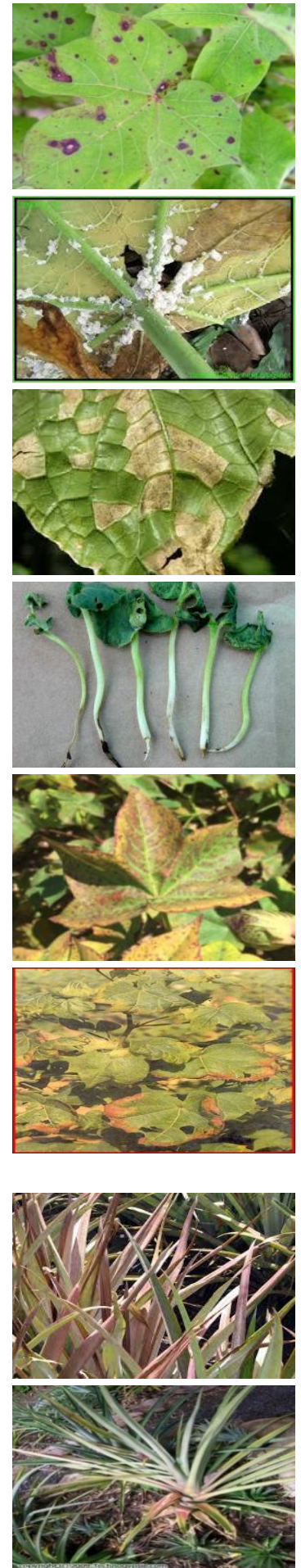
Ralstonia solancearum

Rhizoctonia

Spider mites

Thielaviopsis

Cylindrocladium

Ralstonia solancearum

Rhizoctonia

Spider mites

Thielaviopsis
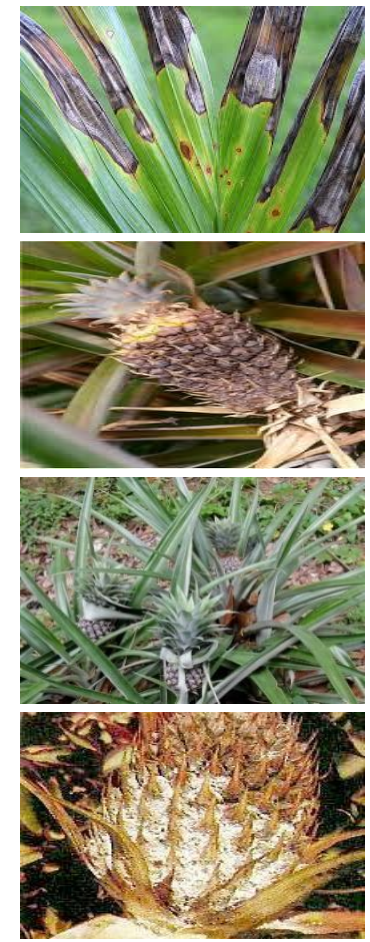

\section{Strawberry}
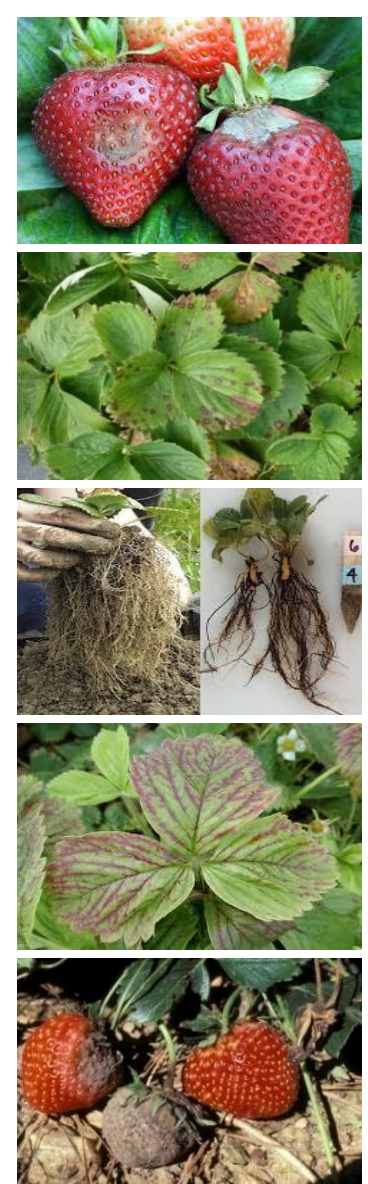
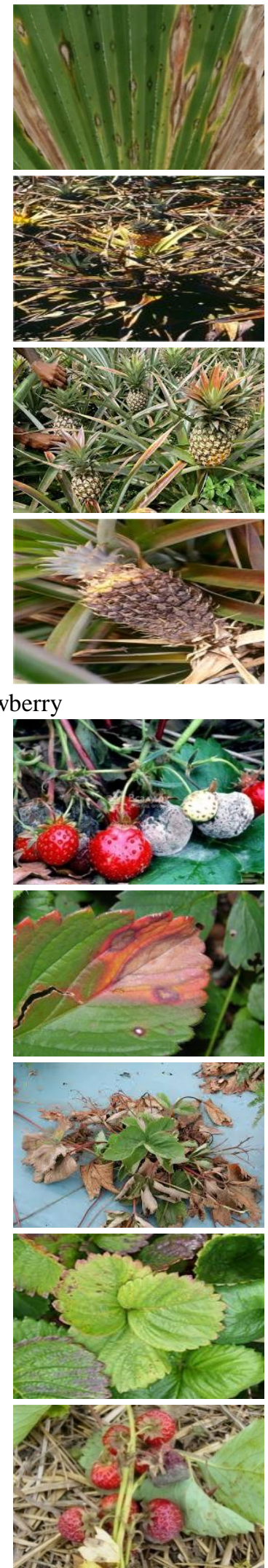
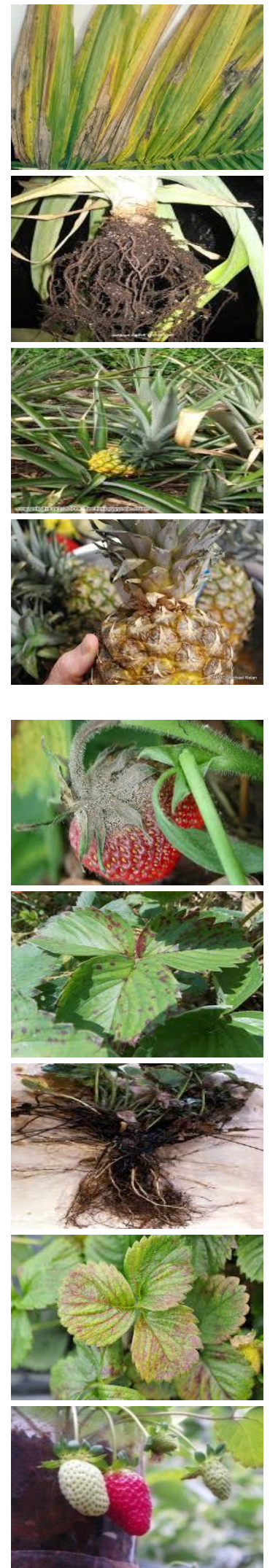

Figure. 3 Sample leaf images for various types of diseases 
Contaminated soil

Damp soil

High humidity soil
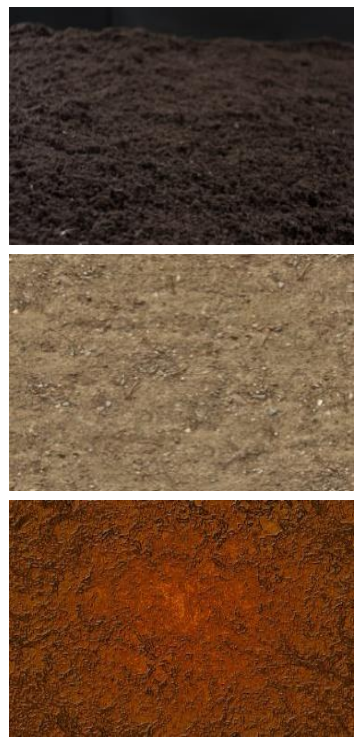

Over watering and high nitrogen soil

Warm, overlay moist soil

Warm and heated soil
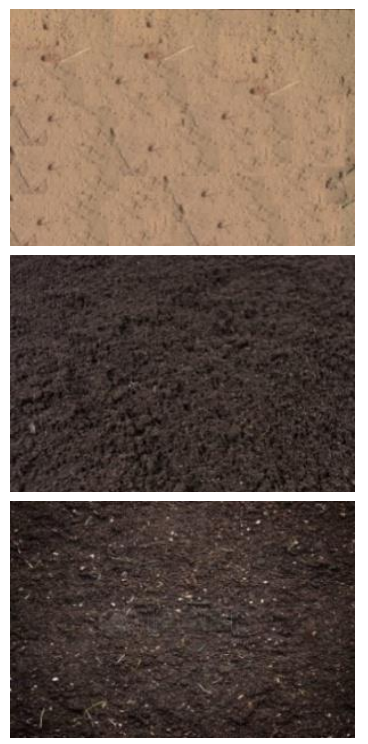
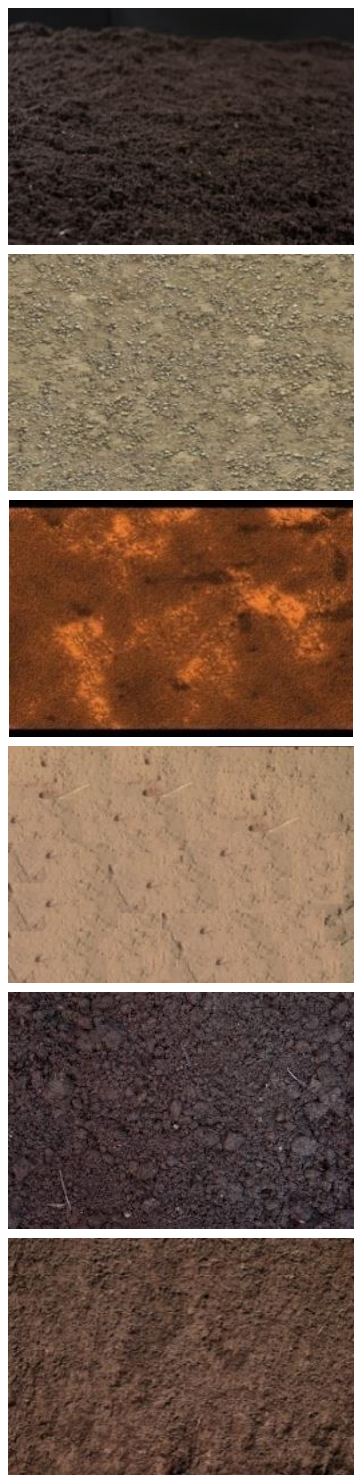

Figure. 4 Sample soil images
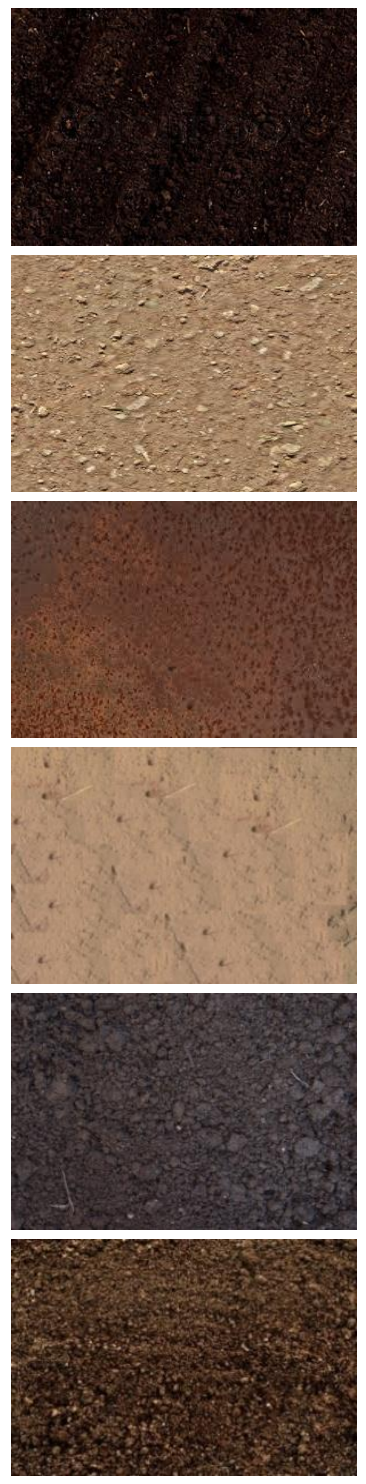

\subsection{Accuracy}

Accuracy is defined as the ratio of true positives and true negatives to the sum amount of all results examined. It is measured as:

$$
A c c=\frac{T P+T N}{T P+T N+F P+F N}
$$

Fig. 5 shows the accuracy of different classification methods for leaf disease prediction. The accuracy of MCNN for cotton leaf disease prediction is $8.59 \%, 6.17 \%, 3.61 \%, 2.99 \%$ and $2.38 \%$ greater than the fuzzy logic, SVM, CNN, ICNN and RFCN methods. Hence, it is proved that the proposed MCNN based leaf disease prediction has high accuracy than all other classification methods for leaf disease prediction.

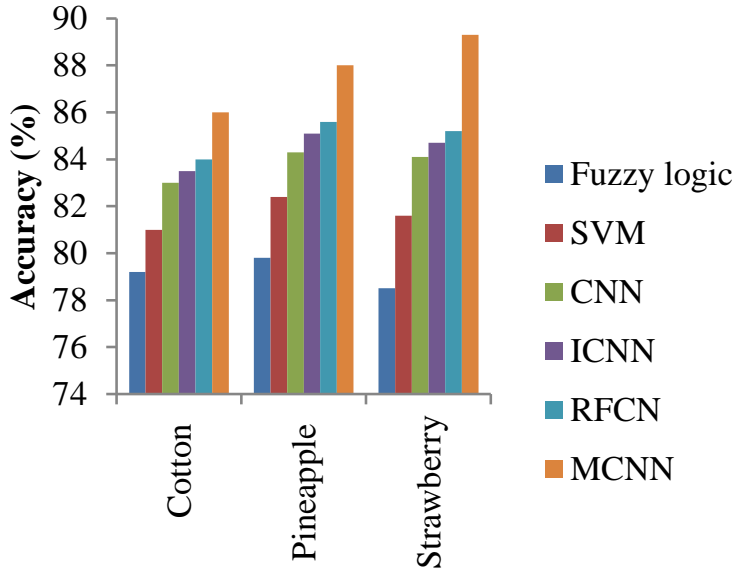

Types of plants

Figure. 5 Comparison of accuracy for leaf disease prediction 


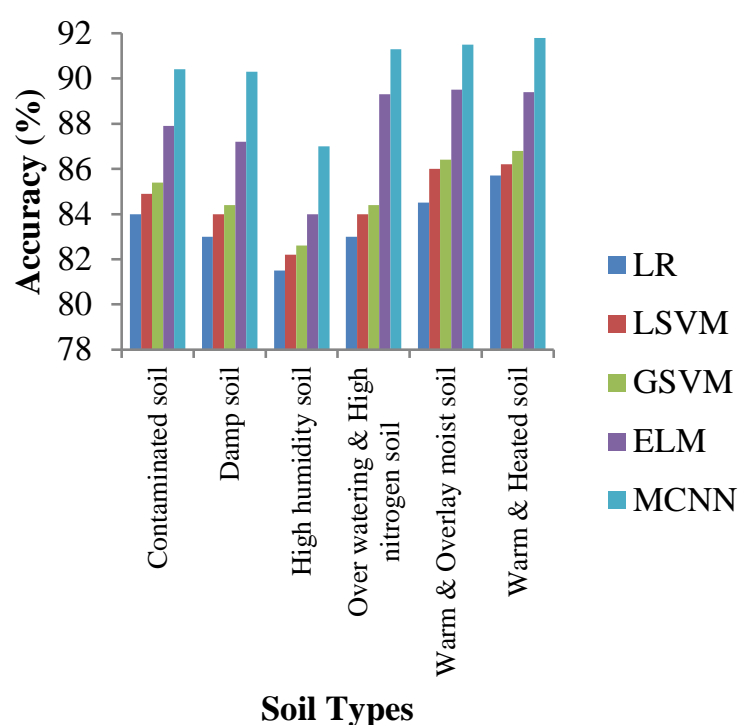

Figure. 6 Comparison of accuracy for soil property prediction

Fig. 6 shows the accuracy of different classification methods for soil property prediction. The accuracy of MCNN for contaminated soil prediction is $7.62 \%, 6.48 \%, 5.85 \%$ and $2.84 \%$ greater than the LR, LSVM, GSVM and ELM methods. Therefore, it is proved that the proposed MCNN based soil property prediction has high accuracy than all other classification methods.

\subsection{Precision}

Precision value is defined as the ratio of predicted features that are relevant and evaluated at the true positive outcomes. It is computed as:

\section{Precision

$$
=\frac{\text { True Positive }(T P)}{\text { True Positive }(T P)+\text { False Positive }(F P)}
$$

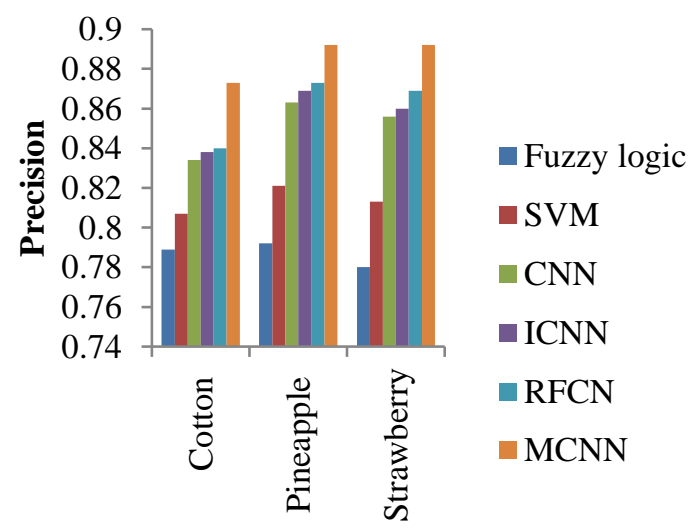

Types of plants

Figure. 7 Comparison of precision for leaf disease prediction

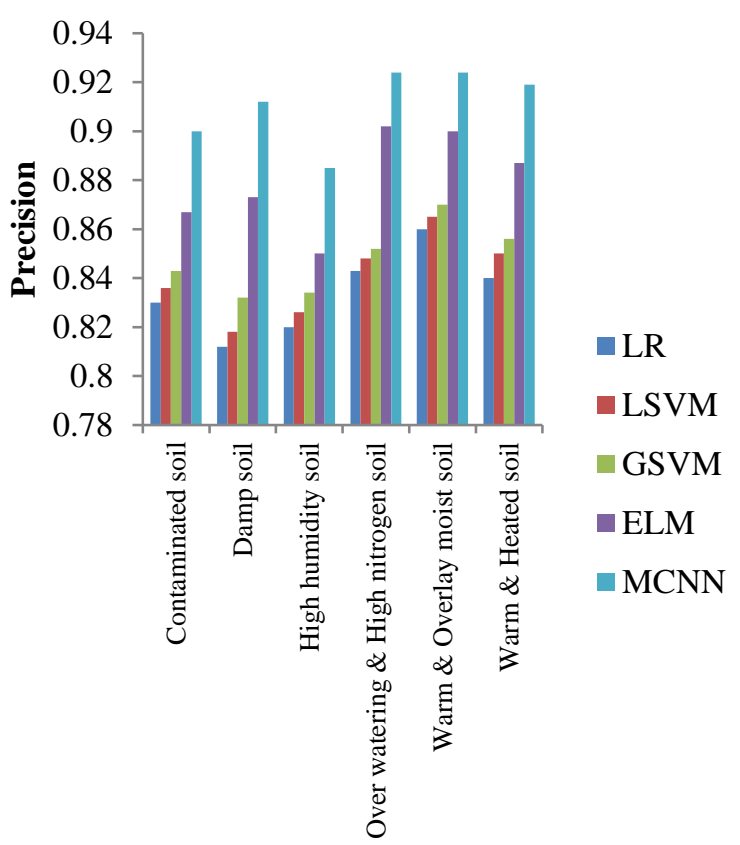

Soil Types

Figure. 8 Comparison of precision for soil property prediction

Fig. 7 shows the precision of various classification methods for leaf disease prediction in terms of precision. The precision of MCNN for cotton leaf disease prediction is $10.65 \%, 8.18 \%$, $4.68 \%, 4.18 \%$ and $3.93 \%$ greater than the fuzzy logic, SVM, CNN, ICNN and RFCN classification methods. Hence, it is proved that the proposed MCNN method has high precision than all other methods for leaf disease prediction.

Fig. 8 shows the precision of different soil property prediction methods. The precision of MCNN for contaminated soil prediction is $8.43 \%$, $7.66 \%, 6.76 \%$ and $3.81 \%$ greater than the LR, LSVM, GSVM and ELM methods. From this analysis, it is proved that the proposed MCNN method has high precision than all other methods for soil property prediction.

\subsection{Recall}

Recall value is calculated based on the prediction value at true positive and false negative predictions.

$$
\text { Recall }=\frac{T P}{T P+\text { False Negative }(F N)}
$$

Fig. 9 shows the recall of various classification methods for leaf disease prediction in terms of precision. The recall of MCNN for cotton leaf disease prediction is $9.61 \%, 7.7 \%, 3.96 \%, 3.09 \%$ 


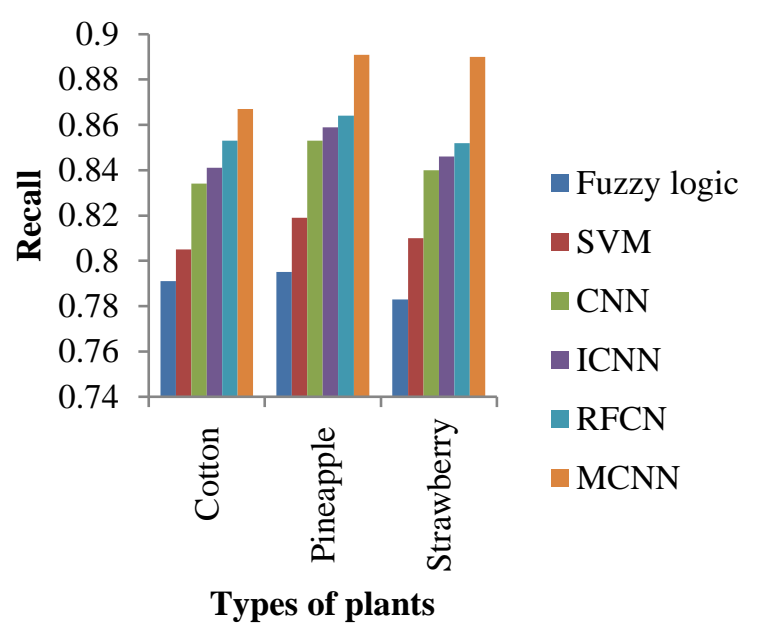

Figure. 9 Comparison of recall for leaf disease prediction

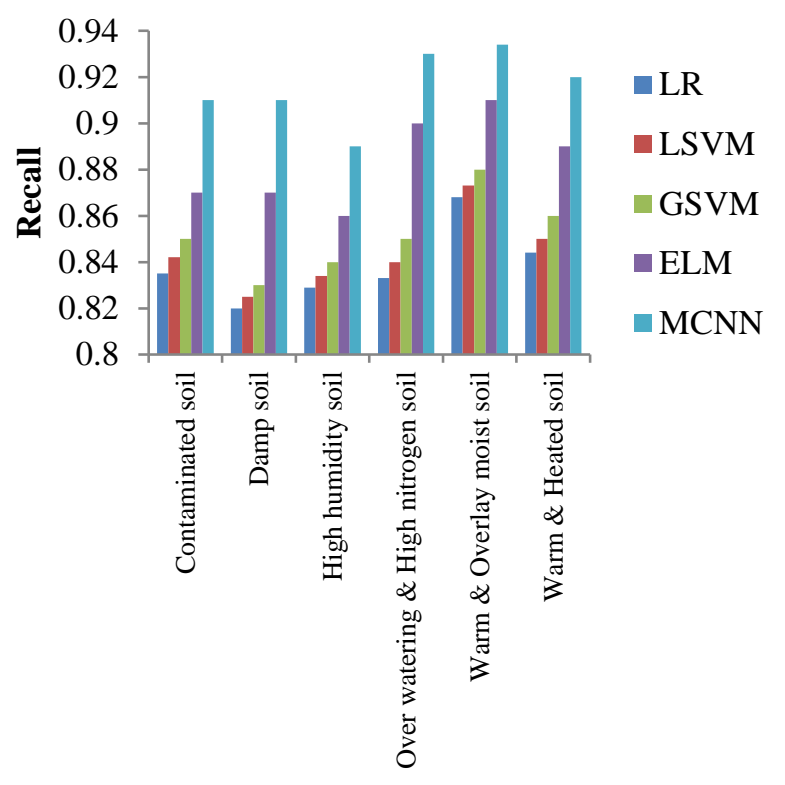

\section{Soil Types}

Figure. 10 Comparison of recall for soil property prediction

and $1.64 \%$ greater than the fuzzy logic, SVM, CNN, ICNN and RFCN classification methods. Hence, it is proved that the proposed MCNN method has high recall than all other methods for leaf disease prediction.

Fig. 10 shows the recall of different soil property prediction methods. The recall of MCNN for contaminated soil prediction is $8.98 \%, 8.08 \%, 7.06 \%$ and $4.6 \%$ greater than the LR, LSVM, GSVM and ELM methods. From this analysis, it is proved that the proposed MCNN method has high recall than all other methods for soil property prediction.

This improvement on efficiency is because of using different channels for both leaf and soil images to simultaneously predict the leaf infections and soil properties.

\section{Conclusion}

In this paper, MCNN is introduced for better prediction of leaf disease and soil property. At first, IOT technology is used to send the captured leaf and soil images to the server. Then, the cellular automata filters are used for noise removal from leaf and soil images. The preprocessed leaf images and soil images are processed in two different channels of $\mathrm{CNN}$ and finally the feature matrices of leaf images and soil images are concatenated for prediction of leaf disease and soil property. MCNN based prediction method ensures the optimum generalizability by concatenating the feature matrices from two different channels for leaf image and soil image. The experimental results proved that the MCNN based leaf disease prediction and soil property prediction has better has better accuracy, precision and recall than the state-of-the-art methods.

\section{Conflicts of Interest}

The authors declare no conflict of interest.

\section{References}

[1] I. Cisternas, I. Velásquez, A. Caro, and A. Rodríguez, "Systematic literature review of implementations of precision agriculture", Computers and Electronics in Agriculture, Vol. 176, p. 105626, 2020.

[2] Y. $\mathrm{Lu}$ and S. Young, "A survey of public datasets for computer vision tasks in precision agriculture", Computers and Electronics in Agriculture, Vol. 178, p. 105760, 2020.

[3] K. T. Dige, "Precision Agriculture in India: Opportunities and Challenges", International Journal of Research in Engineering, Science and Management, Vol. 3, No. 8, pp. 395-397, 2020.

[4] M. Chohan, A. Khan, R. Chohan, S. H. Katpar, and M. S. Mahar, "Plant disease detection using deep learning", International Journal of Recent Technology and Engineering, Vol. 9, No 1, pp. 909-914, 2020.

[5] G. Dhingra, V. Kumar, and H. D. Joshi, "A novel computer vision based neutrosophic approach for leaf disease identification and classification", Measurement, Vol. 135, pp. 782-794, 2019.

[6] X. E. Pantazi, D. Moshou, and A. A. Tamouridou, "Automated leaf disease detection 
in different crop species through image features analysis and One Class Classifiers", Computers and Electronics in Agriculture, Vol. 156, pp. 96-104, 2019.

[7] G. Geetharamani and A. Pandian, "Identification of plant leaf diseases using a nine-layer deep convolutional neural network", Computers \& Electrical Engineering, Vol. 76, pp. 323-338, 2019.

[8] P. Jiang, Y. Chen, B. Liu, D. He, and C. Liang, "Real-time detection of apple leaf diseases using deep learning approach based on improved convolutional neural networks", IEEE Access, Vol. 7, 59069-59080, 2019.

[9] L. X. B. Sorte, C. T. Ferraz, F. Fambrini, R. D. R. Goulart and J. H. Saito, "Coffee leaf disease recognition based on deep learning and texture attributes", Procedia Computer Science, Vol. 159, 135-144, 2019.

[10] M. H. Saleem, S. Khanchi, J. Potgieter, and K. M. Arif, "Image-Based Plant Disease Identification by Deep Learning MetaArchitectures", Plants, Vol. 9, No. 11, 1451, 2020.

[11] A. Nigam, A. K. Tiwari, and A. Pandey, "Paddy leaf diseases recognition and classification using PCA and BFO-DNN algorithm by image processing", Materials Today: Proceedings, Vol. 33, pp. 4856-4862, 2020.

[12] P. K. Sethy, N. K. Barpanda, A. K. Rath, and S. K. Behera, "Deep feature based rice leaf disease identification using support vector machine", Computers and Electronics in Agriculture, Vol. 175, p. 105527, 2020.

[13] J. Sun, Y. Yang, X. He, and X. Wu, "Northern Maize Leaf Blight Detection Under Complex Field Environment Based on Deep Learning", IEEE Access, Vol. 8, pp. 33679-33688, 2020.

[14] M. Kovačević, B. Bajat, and B. Gajić, "Soil type classification and estimation of soil properties using support vector machines", Geoderma, Vol. 154, No. 3-4, pp. 340-347, 2010.

[15] D. Masri, W. L. Woon, and Z. Aung, "Soil property prediction: An extreme learning machine approach", In: Proc. of International Conference on Neural Information Processing, pp. 18-27, 2015. 